HANS-JÜRGEN BECKER, Regensburg

\title{
Die Entwicklung der Appellation im kanonischen Recht: Von der klassischen Periode bis zur nachtridentinischen Epoche
}

\section{Einleitung}

„Ich bekenne, dass die Appellationen für die Welt ein großes und umfassendes Gut sind, das so notwendig ist, wie es die Sonne für das menschliche Leben ist. In der Tat sind sie gewissermaßen wie eine Sonne der Gerechtigkeit, die die Werke der Finsternis beleuchtet und widerlegt. ${ }^{\prime 1}$

Diese Worte richtet Bernhard von Clairvaux (1090-1153) um das Jahr 1148 an Eugen III., also jenen Papst, der einst sein Schüler war. Im zweiten Buch seiner Schrift mit Ratschlägen für eine gute Kirchenleitung geht der Kirchenlehrer auf das Thema der Appellation ein. Doch geht es Bernhard nicht darum, das Appellationswesen der römischen Kurie zu preisen. Ganz im Gegenteil klagt Bernhard den Missbrauch an, der dadurch entstanden ist, dass allzu viele Streitfälle nicht vor Ort entschieden werden, sondern dass aus aller Welt unter Umgehung der örtlichen Instanzen an die römische Kurie appelliert wird. ${ }^{2}$ Er schildert sehr anschaulich, dass in

\footnotetext{
1 „Fateor grande et generale mundo bonum esse appellationes: idque tam necessarium, quam solem ipsum mortalibus. Revera quidem sol justitiae est prodens ac redarguens opera tenebrarum." BERNHARD VON ClaIRVAuX, De consideratione libri V ad Eugenium III., Lib. III, 2.10, insbes. 763.

${ }^{2}$ Hierzu PHILLIPS, Kirchenrecht 220f. (§ 213 „Oberste Gerichtsbarkeit“, 194-310); UlLMANN, Kurze Geschichte 170; SCHIMMELPFENNIG, Das Papsttum 179.
}

der Mitte jenes Jahrhunderts, das die Rechtshistoriker gerne "das juristische Jahrhundert" nennen, der apostolische Stuhl mit Rechtsstreitigkeiten aus allen Teilen der westlichen Christenheit überschüttet wurde, dass die römische Kurie vom Getriebe der im römischen Recht ausgebildeten Advokaten erfüllt war, dass aber wenig vom Gebet, von der Meditation und von einer Haltung zu spüren war, die der Erkenntnis Gottes gewidmet war. So gibt Bernhard in dieser Schrift, die man auch als eine Art von Fürstenspiegel verstehen kann, dem päpstlichen Monarchen Ratschläge, wie das Appellationswesen so geregelt werden kann, dass der Papst als oberste Gerichtsinstanz gleichwohl seine wahre Aufgabe als Monarch erfüllen kann. Diese Ausführungen über den Wert der Appellation nach Rom und über die Gefahren und Unzulänglichkeiten, die mit dem Appellationswesen verbunden sein können, haben viele mittelalterliche Juristen, die sich mit dem Recht der Appellation beschäftigten, angeregt. Noch im Zeitalter von Humanismus und Reformation hielt Melchior Goldast (1578-1635) ${ }^{3}$ diesen Text für so interessant, dass er ihn in seine Textsammlung zur Reichsverfassungsgeschichte aufnahm. ${ }^{4}$ Dies mag ein erster Beleg dafür sein, dass kirchliche Lehren von der Appellation an ein höchstes

\footnotetext{
${ }^{3}$ HOKE, Goldast.

${ }^{4}$ GOLDAST, Monarchia 68-86; vgl. CASPARY, Späthumanismus 95-98.
} 
Gericht, selbst wenn sie im Mittelalter formuliert worden waren, auch noch in der frühen Neuzeit breites Interesse gefunden und Einfluss auf die Entwicklung von weltlichen Rechtsvorstellungen genommen haben.

Bevor die Entwicklung des Appellationsrechts im Folgenden skizzenhaft dargestellt wird, soll zuvor kurz zum Forschungsstand meines Themas berichtet werden. Die allgemeinen Darstellungen, etwa von Feine ${ }^{5}$ oder Plöchl, ${ }^{6}$ sind enttäuschend. Am ergiebigsten sind die älteren Darstellungen von Wilhelm Endemann ${ }^{7}$ und Philipp Hergenröther. ${ }^{8}$ An neueren Monographien stehen die Arbeiten von Heribert Schmitz, ${ }^{9}$ Richard Puza, ${ }^{10}$ Anton Morhard ${ }^{11}$ und Wiesław Litewski ${ }^{12}$ zur Verfügung. Zu hoffen ist, dass Knut Wolfgang Nörr seiner soeben erschienenen Untersuchung über das Erkenntnisverfahren erster Instanz im romanischkanonischen Prozessrecht ${ }^{13}$ einen weiteren Band über das Recht der Appellation folgen lässt.

\section{Zur geschichtlichen Entwicklung der Rechtsquellen}

\subsection{Anfänge in der Spätantike}

Die frühe Kirche formierte sich in einer Gesellschaft, die vom römischen Recht geprägt war. Dass viele rechtliche Institute, die von der Kirche entwickelt wurden, im römischen Recht ihr Vorbild hatten, steht außer Frage. Dies gilt auch

${ }^{5}$ FEINE, Kirchliche Rechtsgeschichte 336f. und 433436.

${ }^{6}$ PLÖCHL, Geschichte des Kirchenrechts 358f.

${ }^{7}$ ENDEMANN, Civilprozessverfahren, insbes. 298-308.

${ }^{8}$ HERGENRÖTHER, Die Appellationen.

9 SCHMITZ, Appellatio extraiudicialis.

${ }^{10}$ PuZA, Res iudicata.

${ }^{11}$ MORHARD, Die gerichtliche Berufung.

${ }^{12}$ LITEWSKI, Appeal; DERS., Der römisch-kanonische Zivilprozeß.

${ }^{13}$ NÖRR, Romanisch-kanonisches Prozessrecht. für die Grundzüge des Appellationsrechts. Aus Zeugnissen des 4. Jahrhunderts wissen wir, dass Bischöfe als erste Instanz in den anliegenden Rechtssachen im Gebiet der Stadt, in der sie ihren Sitz hatten, fungierten. Die zweite Instanz war die Provinzialsynode. So heißt es etwa in can. 5 des 1. Konzils von Nicäa (325), ${ }^{14}$ dass ein Exkommunizierter seinen Streitfall vor die zweimal jährlich tagende Versammlung der Bischöfe der Provinz bringen kann. Die dritte Instanz bildete der Bischof von Rom, der nach can. 3 der Reichssynode von Sardika (343) ${ }^{15}$ als höchste Appellationsinstanz für die von Provinzialsynoden abgesetzten Bischöfe anerkannt wurde.

In den Beschlüssen der 4. Synode von Karthago wurden am Ende des 4. Jahrhunderts Grundzüge eines Instanzenzuges sichtbar: Der angeklagte Kleriker sollte nicht vom Bischof allein, sondern von einem Kollegium von mehreren Nachbarbischöfen verurteilt werden. Jedenfalls wurde eine Berufung gegen das bischöfliche Urteil an die Provinzialsynode allgemein anerkannt. ${ }^{16}$ Freilich kann man zu dieser Zeit noch nicht von einer gefestigten Rechtsbildung sprechen. Im Zusammenhang mit den Streitigkeiten zwischen Rom und der afrikanischen Kirche über die Frage der sog. transmarinen Appellationen wird auf einem Konzil der Satz beschlossen: „Ut nullus ad Romanam ecclesiam audeat appellare."17 Die Ausgestaltung des Appellationswesens dieser Zeit ist kompliziert und noch nicht genügend aufgearbeitet. Im 19. Jahrhundert taten sich Forscher wie Georg Phillips ${ }^{18}$ oder Philipp Hergenröther ${ }^{19}$ leicht, weil sie in

\footnotetext{
${ }^{14}$ Alberigo, Conciliorum oecumenicorum decreta 8.

15 DURST, Sardika 2; BRENNECKE, Rom.

${ }^{16}$ Morhard, Die gerichtliche Berufung 94; HefEle, Conciliengeschichte 2, 74 (Nr. 66).

${ }^{17}$ MORHARD, Die gerichtliche Berufung 95; SCHINDLER, Afrika, insbes. 689; GIRADET, Appellatio.

${ }^{18}$ PHILLIPS, Kirchenrecht 194-310.

${ }^{19}$ HeRgenRÖTHER, Die Appellationen 9.
} 
dieser Epoche gerade Entwicklungslinien ziehen wollten, gestützt auf die Prämisse: „Die Appellation an den Papst ist im göttlichen Recht begründet."

\subsection{Decretum Gratiani (Causa 2 quaestio 6) und die Dekretisten}

Klarer werden die Rechtsverhältnisse im 12. Jahrhundert. Gratian hat um 1140 zum Appellationsrecht in seinem Dekret (Causa 2 quaestio 6) insgesamt 41 canones aus sehr unterschiedlichen Quellen zusammengestellt, die verschiedenen Epochen angehören, angefangen von den Digesten (Dig. 49.1.4; 49.4.5) über Beschlüsse von Konzilien bis hin zu Papstbriefen. Gratian ist sich bewusst, dass die Leitlinien auf das römische Recht zurückzuführen sind, sagt er doch am Ende des Abschnitts: „Haec omnia in VII. lib. Codicis inveneris, a tit. De appellationibus et consultationibus, usque ad tit. Ne liceat in una eademque causa."20

Gratian stellt den Abschnitt unter die Überschrift: "Quo remedio causa viciata sublevetur, et si remedium illud sit dandum ei, qui causa dilationis vocem appellationis exhibuerit? Causa vero viciata remedio appellationis sublevari poterit" ${ }^{21}$

Die causa viciata, die falsch behandelte Sache, kann also mit einer Appellation korrigiert werden. Aber schon in der Überschrift wird das Problem angesprochen, das $\mathrm{zu}$ allen Zeiten mit der Appellation verbunden ist: Kann das Hilfsmittel der Appellation auch von dem in Anspruch genommen werden, der es allein um der Verzögerung willen anwendet? Die bei Gratian folgende Zusammenstellung von einschlägigen

\footnotetext{
${ }^{20}$ Dict. Grat. post C. 2 q. 6 can. 41 (ediert: FrIEDBERG, Corpus iuris canonici 1, 483). Das Zitat verweist auf (Cod. 7.62-Cod. 7.70). Vgl. hierzu JACOBI, Der Prozeß im Decretum Gratiani; RocCA, Il processo in Graziano; MORHARD, Die gerichtliche Berufung 96f.; SCHMITZ, Appellatio extraiudicialis 3-7.

${ }^{21}$ Dict. Grat. ante C. 2 q. 6 c. 1 (ediert: FrIEDBERG, Corpus iuris canonici 1, 466).
}

Rechtsquellen leidet darunter, dass die Terminologie der unterschiedlichen Epochen angehörenden Quellen sehr uneinheitlich ist. Auch sind Widersprüche $\mathrm{zu}$ bemerken. Doch sind die Grundzüge des kirchlichen Appellationswesens, wie Gratian sie sieht, zu erkennen. ${ }^{22}$ Die Appellation dient der Überprüfung einer Rechtssache durch den Oberrichter, unbeschadet einer unmittelbaren Berufung an den Apostolischen Stuhl. Etwas pathetisch heißt es in can. 8: "Ad Romanam ecclesiam ab omnibus, maxime tamen ab obpressis est appellandum, et concurrendum quasi ad matrem, ut eius uberibus nutriantur, auctoritate defendantur, a suis obpressionibus releventur, quia non potest nec debet mater oblivisci filium suum. “23

Die Appellation kann im Unterschied zum römischen Recht nicht nur gegen ein Urteil, sondern schon vor Fällung des Urteils eingelegt werden. Die Appellation hat zwei Wirkungen: Zum einen wirkt sie devolutiv, so dass die Entscheidungsbefugnis auf den Oberrichter übergeht, zum anderen hat sie einen Suspensiveffekt, der bewirkt, dass das Urteil nicht rechtskräftig wird und somit auch nicht vollstreckt werden kann.

Soweit die Appellation gegen ein Urteil eingelegt wird, muss dies innerhalb von zehn Tagen geschehen, in der Regel schriftlich. Allerdings muss der Appellant dartun, dass er sich ungerecht beschwert fühlt. Sowohl für die Appellation gegen ein Urteil wie für die Appellation vor einem Urteil bietet Gratian Formulare an. Auch für die innerhalb von 30 Tagen auszufertigenden sog. apostoli oder litterae dimissoriae, also das Überweisungsschreiben, das der Richter der unteren Instanz innerhalb von 30 Tagen an den Oberrichter zu senden hat, bietet Gratian ein Formular an.

\footnotetext{
22 Das Folgende nach ScHMITZ, Appellatio extraiudicialis 3-22.

${ }^{23}$ Decr. Grat. C. 2 q. 6 c. 8 (FrIEDBERG, Corpus iuris canonici $1,467 f$.).
} 
Die Rechtsquellen, die Gratian zusammengestellt und mit Hinweisen versehen hat, sind umfangreich, doch bleibt vieles unklar. Schon Stephan von Tournai (1135-1203) hat bemängelt, dass Magister Gratian das Appellationsrecht "non solum diffuse, sed etiam confuse" behandelt habe. ${ }^{24}$ Das gab der aufblühenden Schule der Dekretistik Gelegenheit, in ihren großen Summen zum Dekret eine geschlossene Systematik $\mathrm{zu}$ entwickeln. Eine besondere Rolle spielt daneben eine eigene Literaturgattung zum kirchlichen Prozessrecht, die ordines iudiciorum bzw. ordines iudiciarii. ${ }^{25}$ Hier wurden im 12. und 13. Jahrhundert in einer Kombination von römischem Recht und zeitgenössischem päpstlichen Dekretalenrecht die practica iuris wissenschaftlich aufgearbeitet und eine Wissenschaft vom Prozessrecht begründet. Das einflussreiche Werk "Speculum iuris" des Guilielmus Durantis (um 1230-1296) hat diese Wissenschaft am Ende des 13. Jahrhunderts auf hohem Niveau zusammengefasst. ${ }^{26}$

\subsection{Das 3. Laterankonzil von 1179 (can. 6)}

Die auf Gratian folgenden Jahre sind durch das starke Anwachsen der Rechtsquellen in Gestalt von päpstlichen Dekretalen und von Konzilsbeschlüssen charakterisiert. Unter den Dekretalen ist besonders jene von Alexander III. „Cum sit sancta (bzw. sacrosancta) Romana ecclesia “27 von 1171/72 von Bedeutung, in der der Papst auf Anfrage des Erzbischofs von Reims wichtige Regelungen für die Appellation aufstellt. ${ }^{28}$ Hier ist zunächst von der Frist zur Verfolgung oder

\footnotetext{
${ }^{24}$ StePHAN VON TOURNAI, Summe über das Decretum Gratiani 172f. (ad. C. 2 q. 6 pr.).

${ }^{25} \mathrm{Zu}$ dieser Literaturgattung FOWLER-MAGERL, Ordo.

${ }^{26} \mathrm{Vgl}$. LEPSIUS, Durantis, Guilelmus.

${ }^{27}$ Liber Extra 2.28.5 (ediert: FRIEDBERG, Corpus iuris canonici 2, 411).

${ }^{28}$ SCHMITZ, Appellatio extraiudicialis 25f.; NÖRR, Ein Baustein, insbes. 469-471.
}

Einführung der Appellation in einem Gerichtsverfahren die Rede, die auf ein Jahr festgesetzt wird, auf Antrag aber bis zu zwei Jahre verlängert werden kann. Lässt der Appellant diese Frist verstreichen, wird das Urteil des Unterrichters rechtskräftig. Im Hinblick auf Appellationen, die außerhalb eines Prozesses erfolgen (appellationes a gravamine et ante litis ingressum, appellationes extraiudiciales) - zum Beispiel bei Beschwer durch eine Wahl oder die Besetzung eines Benefiziums -, wird angeordnet, dass sie im Unterschied zum römischen Recht - grundsätzlich als zulässig angesehen werden. Sie sind an den Oberrichter zu richten.

Für das Recht der Appellation ist vor allem can. 6 des unter Alexander III. tagenden 3. Laterankonzils von 1179 von Bedeutung. In den einleitenden Worten zu diesem can. „Reprehensibilis" ${ }^{29}$ wird deutlich, dass - wie auch bei Bernhard von Clairvaux nachzulesen ist - das Appellationswesen in eine Krise geraten war. Zum einem versuchen manche Bischöfe und Archidiakone, jene Personen, die gegen ihre Entscheidungen Berufung einlegen wollen, durch Suspension und Exkommunikation daran zu hindern. Auf der anderen Seite versuchen viele, denen eine Zurechtweisung durch ihre Vorgesetzten droht, ohne einen legitimen Beschwerdegrund wahllos Appellationen einzulegen: "Zur Verteidigung des Unrechts missbrauchen sie so, was zum Schutz Unschuldiger eingerichtet worden ist. “30 Das Konzil geht gegen beide Arten des Missbrauchs - sowohl gegen die Behinderung von rechtmäßigen Appellationen als auch die inflationäre Einlegung von Appellationen - vor. Was den Missbrauch der Appellationen angeht, so verfügt das Konzil, dass gegen Maßnahmen der Kirchenzucht außerhalb eines

\footnotetext{
${ }^{29}$ Alberigo, Conciliorum oecumenicorum decreta 214 = Liber Extra 2.28.26 (ediert: FRIEDBERG, Corpus iuris canonici 2, 418f.).

30 "Ad defensionem iniquitatis usurpant, quod ad praesidium innocentium dignoscitur institutum."
} 
Prozesses (ante ingressum causae) grundsätzlich nicht appelliert werden kann. Nur ausnahmsweise kann Berufung eingelegt werden, wenn der Betroffene meint, ohne Appellation nicht auskommen zu können. In diesem Fall wird ihm eine Frist gesetzt. Erscheint der Appellant jedoch nicht zum Termin, so ist er verpflichtet, dem Gegner seine Kosten zu ersetzen. Mit der Ausnahmebestimmung wird deutlich, dass die Möglichkeit, gegen eine Beschwernis Berufung auch außerhalb eines Prozesses einzulegen, nicht generell beseitigt werden sollte.

\subsection{Liber Extra (2.28); Liber Sextus (2.15), Clementinae (2.12) und die Dekretalisten}

Sowohl die genannte Dekretale „Cum sit sancta Romana" (X 2.28.5) wie auch die Konzilsnorm "Reprehensibilis" (X 2.28.26) gehören zu jenen canones, die in den Liber Extra von 1234 aufgenommen worden sind. Besonders reichhaltigen Stoff boten auch die Beschlüsse des 4. Laterankonzils von 1215, von denen drei dem Appellationswesen gewidmet sind. So wird in can. 35 "Ut debitus"31 das Recht, schon vor Urteilsverkündung Berufung einzulegen, dadurch beschränkt, dass der Appellant in Zukunft hierfür einen vernünftigen Grund angeben muss. Kommt der Oberrichter zum Ergebnis, dass ein solcher Grund nicht vorliege, wird die Sache an den Unterrichter zurückverwiesen, wobei der erfolglose Appellant die Kosten des Gegners zu tragen hat.

Raimund von Peñafort (1175-1275) hatte bekanntlich 1234 eine systematische Sammlung des kirchlichen Rechts angelegt. In Buch 2 dieses Liber Extra wurde das Prozessrecht aufgenommen, wobei das Appellationsrecht im Unter-

\footnotetext{
${ }^{31}$ Alberigo, Conciliorum oecumenicorum decreta 251

= Liber Extra 2.28.59 (ediert: FRIEDBERG, Corpus iuris canonici 2, 437). Zum Folgenden vgl. MORHARD, Die gerichtliche Berufung 101-106.
}

titel 28 Aufnahme fand. Die später folgenden Kodifikationen des kirchlichen Rechts, der Liber Sextus (1298) und die Clementinae (1317), weisen gleichfalls jeweils innerhalb des Prozessrechts einen eigenen Titel „De appellationibus" auf. Die hier zusammengestellten Rechtsquellen haben das Recht der Appellation sowohl in der kirchlichen wie auch in der weltlichen Gerichtsbarkeit über lange Jahrhunderte geprägt.

\subsection{Reformkonzilien des}

\section{Jahrhunderts}

Gleichwohl wurde das Appellationswesen immer wieder zum Stein des Anstoßes. Bezeichnend sind etwa die wiederholten Klagen über einen um sich greifenden Missbrauch. Auf dem Konzil zu Pisa wird im Juli 1409 etwa als Reformwunsch formuliert: „Und weil keine Gelegenheit zum Unrechttun dort entstehen soll, woher das Recht kommt, legen die Genannten (Vertreter der Nationen) demütig bittend die Klage vor, dass die Römischen Bischöfe sich seit mehreren Jahren einige Verfahren zur Prüfung und Entscheidung an der Römischen Kurie vorbehalten haben, obgleich sie ihrer Natur nach und gemäß dem gemeinen Recht in den betreffenden Gebieten hätten vorgelegt, geprüft und entschieden werden müssen. Dies führt zur Verwirrung der kirchlichen Gerichtsbarkeit $[\ldots]^{\prime \prime}{ }^{32}$ Die Synode bittet dringend darum, der Papst möge das abstellen. Es geschieht aber nichts.

So muss die Thematik auf dem Konzil von Konstanz erneut verhandelt werden. Ein kleiner

32 "Et quia non debet nasci iniuriarum occasio, unde iura nascuntur, conquerendo exponunt humiliter supplicantes predicti, quod Romani pontifices ab aliquibus annis citra quaslibet causas ad ipsos per simplicem querelam vel appellacionem quamlibet, que de sui natura et de iure communi deberent committi, examinari et decidi in partibus, commiserunt examinandas et decidendas in curia Romana. Quod cedit in turbacionem iurisdictionis ecclesiastice [...]". Zit.n. MieThKe, WeINRICH, Quellen zur Kirchenreform 1, 174-175. 
Niederschlag findet sich im Konkordat Martins V. mit den Konzilsnationen vom 21. März 1418, wo in Abschnitt IV zugesagt wird, dass Rechtsstreitigkeiten, die nach Recht und Herkommen nicht vor ein kirchliches Gericht gehören, nicht von der Römischen Kurie angenommen werden. ${ }^{33}$ Freilich hat diese Zusage nicht viel an den Missbräuchen geändert. Jedenfalls wird auf dem Konzil von Pavia/Siena 1423 durch die französische Nation erneut in Kapitel 19 vorgetragen: „Viele Kirchenleute, besonders die einfachen und die in weit entfernten Gegenden, werden sehr belastet, vielen Gefahren ausgesetzt und oft dadurch ihrer Rechte beraubt, dass alle Prozesse und alle Appellationen ohne Berücksichtigung der mittleren und unmittelbaren Gerichtshöfe an die Römische Kurie gebracht und dort verhandelt werden; daraus entstehen viele Gefahren und Unzuträglichkeiten in der Kirche Gottes, viele gehen dadurch ihrer rechtmäßigen Titel verlustig und verlieren ihre Prozesse [... "..34 Wiederum bittet man um Abhilfe und wieder geschieht nichts.

Das Basler Konzil hat in seiner 31. sessio am 24. Januar 1438, also nach der Verlegung des Konzils nach Ferrara, im Dekret „Ecclesiasticae solicitudinis" angeordnet, dass von nun an alle kirchlichen Streitigkeiten - mit Ausnahme der causae maiores und der Streitigkeiten wegen Besetzung der Bistümer und Abteien - in allen Gegenden, die über vier Tagreisen von der römischen Kurie entfernt seien, in diesen Gebieten selbst durch die gewöhnlichen Richter entschie-

\footnotetext{
${ }^{33}$ Ebd. 1, 523.

34 "Item quia multi ecclesiastici et presertim simplices illi, qui sunt de longinquis partibus, nimis gravantur atque periculis multis exponuntur et frequenter perdunt jura sua eo, quod omnes cause et omnes appellaciones, non observatis quibuscumque mediis aut judicibus immediatis, introducuntur et tractantur in curia Romana, sequanturque ex hoc pericula et inconveniencia multa in ecclesia dei, multi per hoc suis privantur titulis justis et causis [...]". Zit.n. ebd. 2, 126-127.
}

den werden sollten. ${ }^{35}$ Glaube jemand, er sei durch einen richterlichen Spruch beschwert worden, so könne er an den höheren Kirchenobern, zuletzt auch an den Papst appellieren, aber der Instanzenweg müsse eingehalten werden. Werde im Instanzenzug nach Rom appelliert, so solle die Sache in der Regel Richtern in partibus übertragen werden. Nur Kardinäle, Vizekanzler, Kämmerer, Großpönitentiare, Protonotare und Offiziale der römischen Kirche seien davon ausgenommen. Das Dekret „Ecclesiasticae solicitudinis" wurde am 30. Juni 1438 durch die Pragmatische Sanktion von Bourges gutgeheißen und für Frankreich übernommen. ${ }^{36}$ Auch die Mainzer Akzeptation vom 26. März 1439 übernahm das genannte Dekret „De causis et appellationibus" des Basler Konzils. ${ }^{37}$ Zugleich wurde dem Konzil von Ferrara/Florenz im Rahmen eines in den Papieren von Nikolaus von Kues überlieferten Reformvorschlags ${ }^{38}$ die Dringlichkeit einer Verbesserung des Appellationswesens vor Augen gestellt. ${ }^{39} \mathrm{Da}$ aber einerseits das Rumpfkonzil von Basel schließlich im Sande verlief und da andererseits das Konzil von Ferrara/Florenz die Thematik nicht aufgriff, wurde im Bereich der römischen Kurie nichts davon verwirklicht.

\subsection{Konzil von Trient (sess. 13, De reform. can. 1-3; sess. 22, De reform. can. 7; sess. 24, De reform. can. 20)}

So musste die Reform des Appellationswesens, die durch die Reformkonzilien nicht bewirkt werden konnte, mehr als 100 Jahre später durch

\footnotetext{
${ }^{35}$ Dekret "Ecclesiasticae solicitudinis", MANSI, Sacrorum conciliorum [...] collectio 159-160 und MiETHKE, WEINRICH, Quellen zur Kirchenreform 2, 380-385; HefEle, Conciliengeschichte 7, 661f.

${ }^{36}$ MiethKe, WeinRich, Quellen zur Kirchenreform 2, 412-441, insbes. 430-431.

${ }^{37}$ Ebd. 2, 442-449, insbes. 447 (Nr. 9).

${ }^{38}$ Hierzu DanNENBAUER, Ein deutscher Reformantrag.

${ }^{39}$ MiETHKE, WeinRich, Quellen zur Kirchenreform 2, 452-457, insbes. 454-455 (Nr. 2).
} 
das Konzil von Trient erneut in Angriff genommen werden. ${ }^{40}$ Dies geschah in der 13 . Sitzung am 11. Oktober 1551 (Reformdekret can. 1-3), ${ }^{41}$ in der 22. Sitzung am 17. September 1562 (Reformdekret can. 7) $)^{42}$ und in der 24. Sitzung am 11. November 1563 (Reformdekret can. 20).43 Hier wird u.a. zum wiederholten Male festgelegt, dass das bischöfliche Gericht die erste Instanz bildet. Jeder Prozess ist in jeder Instanz innerhalb zweier Jahre zu Ende zu führen. Die Appellation ist allerdings ab sofort nur noch gegen Endurteile und jene Interlokute zulässig, die prozessbeendende Wirkung haben. Reformerische Maßnahmen der Bischöfe zur Verbesserung der Sitten dürfen nicht mehr durch eine appellatio frivola unterlaufen werden. Iudex ad quem ist der Metropolit oder dessen Generalvikar. Der Appellant hat diesem die erstinstanzlichen Akten vorzulegen, die ihm vom iudex a quo innerhalb von 30 Tagen zur Verfügung gestellt werden müssen.

\subsection{Codex iuris canonici/1917 (can. 1879-1891)}

Der Codex iuris canonici von 1917 hat das Appellationsrecht nicht grundsätzlich verändert; viele Normen stellen nur gekürzte Fassungen jener canones dar, die bereits im Liber Extra und im Liber Sextus enthalten waren. ${ }^{4}$ Die herausragende Stellung des Papstes als oberster Gerichtsherr wird - unter Bezug auf die Beschlüsse des 1. vatikanischen Konzils - besonders betont.

\footnotetext{
${ }^{40}$ Zum Folgenden vgl. HergenRÖther, Die Appellationen 14f.; SÄGMÜLLER, Lehrbuch 323f.; MORHARD, Die gerichtliche Berufung 106-108.

${ }^{41}$ AlBerigo, Conciliorum oecumenicorum decreta $699 \mathrm{f}$.

${ }^{42}$ Ebd. $739 f$.

${ }^{43}$ Ebd. 772f.

${ }^{44} \mathrm{Vgl}$. die Quellennachweise in den Fußnoten der Ausgabe von GASPARRI, Codex iuris canonici 526-530.
}

\section{Das System der Appellation im kanonischen Prozess}

\subsection{Arten der Berufung}

Nach kanonischem Recht war die Berufung sowohl gegen gerichtliche Entscheidungen (appellatio iudicialis) wie auch gegen eine durch Rechtshandlungen und Beschlüsse herbeigeführte Beschwernis außerhalb eines Prozesses (appellatio extraiudicialis) möglich. ${ }^{45}$ Die im Mittelalter mögliche Berufung gegen Zwischenurteile wurde durch das Konzil von Trient beseitigt.

Um missbräuchliche Appellationen zu vermeiden, wurde ein umfangreicher Katalog von Fällen zusammengestellt, in denen die Appellation nicht zulässig war. Den Studenten gab man als Gedächtnishilfe folgenden Merkvers an die Hand:46

"Súblimís judéx, scelús, execútio, páctum,

Cóntemptús, et rés minimaé, dilátio núlla,

Claúsula, quaé removét, res, quaé notoria cónstat,

Ét textús jurís clarús, posséssio, fátum."

Es war also in folgenden Fällen keine Appellation zugelassen:

1. gegen Urteile des höchsten Richters; in Kriminalfällen bei Vorliegen von Geständnis und Beweis; gegen Vollstreckungsmaßnahmen; bei vertraglich vereinbartem Schiedsgericht,

2. bei Verzicht auf Appellation; bei contumatia; bei Geringfügigkeit der Streitsache; bei Sachen, die keinen Aufschub dulden, haben Appellationen jedenfalls keine Suspensivwirkung,

3. bei Nichtzulassung durch Papst oder Konzil; Ausschluss der Appellation gegen ein Notorium,

\footnotetext{
${ }^{45}$ SCHMITZ, Appellatio extraiudicialis; NÖRR, Ein Baustein.

${ }^{46}$ HeRgENRÖTHER, Die Appellationen 22.
} 
4. bei bloßer Anwendung des Gesetzes; bei possessorischen Klagen (Verweis auf Petitorium); bei Vorliegen von zwei gleichlautenden Erkenntnissen kann nicht zum dritten Mal appelliert werden.

Bei der herausragenden Stellung des Papstes als oberstem Gerichtsherrn versteht es sich, dass eine Appellation gegen ein päpstliches Urteil nicht zulässig ist. ${ }^{47}$

\subsection{Konstitutive Elemente des Appellationsrechts}

Im Folgenden soll die rechtliche Ausgestaltung des Appellationsrechts nach dem Recht der Dekretalen (Liber Extra 2.28; Liber Sextus 2.15, Clementinae 2.12) sowie den Bestimmungen des Konzils von Trient skizziert werden. ${ }^{48}$ Man unterscheidet im Appellationsverfahren drei Schritte: Die Einlegung (interpositio appellationis), die Einführung (introductio appellationis) und die Ausführung (prosecutio oder justificatio appellationis).

Die Appellation ist in der Regel vor dem judex a quo einzulegen. Diese interpositio appellationis kann schriftlich oder mündlich innerhalb einer Frist von zehn Tagen geschehen. Die Appellation ist, wenn keine Ausnahmetatbestände vorliegen, vom judex a quo zuzulassen (deferre appellationi, appellationem recipere). Im Zweifel muss der Richter die Appellation propter reverentiam superioris iudicis zulassen. Der Appellant bittet nun den Richter um die Ausstellung der Apostel (apostoli, libelli dimissorii). In diesem Schriftstück informiert der Unterrichter den Oberrichter über den Prozess und die eingelegte Berufung. Die

\footnotetext{
${ }^{47}$ BECKER, Die Appellation.

${ }^{48}$ Grundlage der folgenden Skizze bilden die Darstellungen von LANCELOTTUS, Institutiones iuris canonici, Lib. III, Tit. VII, 362-377; FERRARIS, Appellatio; ENDEMANN, Civilprozessverfahren; HERGENRÖTHER, Die Appellationen 33-46; SÄGMÜLLER, Lehrbuch 340-346; AmanieU, Appel.
}

Apostel sollen in der Regel in einer Frist von 30 Tagen erteilt werden.

Nunmehr folgt die Einbringung der Appellation (introductio appellationis) vor dem Oberrichter und damit der Beginn des Appellationsprozesses. Zur Durchführung steht dem Appellanten eine Frist von einem Jahr zur Verfügung. Ausnahmsweise kann dem Appellanten eine Frist von zwei Jahren eingeräumt werden. Wird diese Frist versäumt, so gilt die Appellation als verlassen (appellatio deserta). In einer Voruntersuchung prüft der judex ad quem seine Zuständigkeit, die Berechtigung des Appellanten zur Appellation, die gesetzliche Zulässigkeit der Appellation und die Einhaltung der Fristen. Wird die Appellation nicht angenommen, so geht die Sache wieder zurück an den Unterrichter. Andernfalls wird das Hauptverfahren eröffnet. Dazu sind dem Richter die früheren Akten vorzulegen.

Der Appellant hat eine Rechtfertigungsschrift (libellus gravaminum, prosecutio oder justificatio appellationis) vorzulegen, in der er darlegt, inwiefern er sich durch das Urteil der unteren Instanz beschwert glaubt und welche Tatsachen und Beweismittel nach seiner Auffassung bei richtiger Bewertung zu dem durch den Appellationsantrag bezeichneten Ergebnis führen würden. Die Rechtfertigungsschrift muss dem Prozessgegner in einer angemessenen Frist in Abschrift mitgeteilt werden, damit er darauf eine Entgegnung formulieren kann. Sowohl die Rechtfertigungsschrift des Appellanten wie auch die Entgegnung seines Gegners können ein neues Beweisverfahren notwendig machen.

Der Appellationsrichter entscheidet nicht nur, ob gut oder schlecht appelliert worden ist, sondern entscheidet - wenn die Appellation begründet ist - über den Bestand des angefochtenen Urteils. Dieses kann zu Gunsten des Appellanten aufgehoben und durch einen günstigen Richterspruch ersetzt werden (bene appellatum, male judicatum). War dagegen das vorinstanzliche Urteil zutreffend, so bestätigt der Oberrichter das Urteil (bene judicatum, male appellatum). In 
seiner Entscheidung kann der Appellationsrichter auch zu Gunsten des Appellationsgegners und zum Nachteil des Appellanten (in pejus) das Vorurteil abändern.

\subsection{Rechtswirkungen}

Ziel der Appellation ist eine Abänderung des Urteils, durch das sich der Appellant beschwert glaubt. Für die Praxis bedeutsam sind aber auch zwei Wirkungen, die eine Appellation nach sich ziehen kann: der Suspensiv- und der Devolutiveffekt. Bei einer Berufung gegen ein Endurteil besteht der Suspensiveffekt darin, dass der judex a quo die Exekution seines Urteils einstellen muss. Falls dies der Richter unterlässt, kann der judex ad quem auf Antrag des Appellanten die Vollstreckungsmaßnahmen aufheben. Bei einem Zwischenurteil ${ }^{49}$ tritt der Suspensiveffekt erst ein, wenn der Oberrichter die Appellation als begründet anerkannt hat. Der Devolutiveffekt besteht darin, dass bei Appellation gegen ein Endurteil die Jurisdiktion des judex a quo erlischt und die Zuständigkeit für den Streitfall an den Oberrichter übergeht.

\subsection{Probleme der Praxis}

So unverzichtbar das Rechtsmittel der Berufung auch für die Rechtspflege ist, so bringt es doch auch eine Reihe von Nachteilen mit sich. Es war Ziel einer stetig nachgebesserten Gesetzgebung, diese Nachteile einzugrenzen, doch ist dies nicht durchweg gelungen. Durch die Jahrhunderte hindurch wird darüber geklagt, dass durch die Appellation zum einen eine Verzögerung des gerichtlichen Verfahrens eintrete und dass zum anderen mit diesem Rechtsmittel hohe Kosten verbunden seien. Diese Klagen beziehen sich vor allem auf die Konzentration der Appellationsverfahren in Rom, wo durch die erforderlichen langen Reisen und Postwege, durch die Anstellung von spezialisierten Anwälten und

\footnotetext{
${ }^{49}$ Vgl. hierzu SCHLINKER, Die prozessuale Funktion.
}

durch die an der römischen Kurie fälligen Gebühren - unter anderem für die Reinschrift des Urteils, seine Registrierung und Ausfertigung erhebliche Geldmittel eingesetzt werden müssten.

\section{Die Wirkung des Appellations- rechts für die Rechtskirche: Licht und Schatten}

\subsection{Der Ausbau der kirchlichen Gerichtsbarkeit}

Für die Rechtsgeschichte Europas ist die im kirchlichen Bereich zuerst entwickelte umfassende Gerichtsorganisation wegbereitend gewesen. ${ }^{50}$ Schon früh wurde hier ein klarer Instanzenzug entwickelt, an dessen Spitze die päpstlichen Gerichte in Rom standen: zunächst die Sacra Rota Romana ${ }^{51}$ und die Signatura Apostolica, ${ }^{52}$ sodann die Kardinalskongregationen ${ }^{53}$ und schließlich als oberster Richter der Papst. Dieses System, das einen breiten Rechtsschutz garantierte, war allerdings gefährdet. Es waren insbesondere die Regional- und Nationalkirchen, die gegen die starke Zentrierung der Gerichtsbarkeit in Rom Widerstand leisteten. Die Wirksamkeit der kirchlichen Jurisdiktion wurde sodann im Laufe der Neuzeit dadurch gemindert, dass eine Reihe von Territorien sich der Reformation zuwandte und somit aus der Zuständigkeit der

\footnotetext{
${ }^{50}$ LANDAU, Die Anfänge der Prozessrechtswissenschaft.

${ }^{51}$ EMERIX, Tractatus (Tit. 26, 111-114: De appellationibus); Hinschius, Das Kirchenrecht (§§ 44-47: Die Justizbehörden (Tribunale) der Kurie, 392-420); SCHNEIDER, Die Römische Rota; RE, La Curia Romana 226-242; BECKER, Die Sacra Rota Romana; DERS., Kardinal Giovanni Battista de Luca; KillermanN, Die Rota Romana.

52 PUZA, Signatura iustitiae; DERS., Rescriptum; RE, La Curia Romana 212-225.

${ }^{53}$ RE, La Curia Romana 93-196 und 321-434.
} 
römischen Gerichtsbarkeit ausschied. Aber auch in den katholischen Staaten war eine deutliche Tendenz zu spüren, im Sinne eines absolutistischen Staatskirchentums $\mathrm{zu}$ erreichen, dass keine Appellationen an Gerichte außerhalb des eigenen Staates vorgenommen wurden.

\subsection{Zur Gerichtsbarkeit der delegierten Richter und der Ständigen Nuntiaturen}

In Ergänzung der iurisdictio ordinaria entwickelte sich im Mittelalter eine Delegationsgerichtsbarkeit. ${ }^{54}$ Auf der Grundlage des Dekretalenrechts war es möglich, sich unter Umgehung des normalen Instanzenzuges unmittelbar an die Kurie $\mathrm{zu}$ wenden. Wurde dem Antrag entsprochen und mit päpstlichem Justizbrief eine iurisdictio delegata zugestanden, so war die Appellation ausgeschlossen. In den päpstlichen Urkunden wurde dann die Formel appellatione remota verwandt. Wie erfolgreich diese Einrichtung war, ist in der Forschung umstritten. Jedenfalls entstand mit der Delegationsgerichtsbarkeit den römischen Gerichten eine Konkurrenz. Das Drängen der Nationalstaaten führte aber zu einer Dezentralisierung der kirchlichen Rechtsprechung: Die sog. Konkordate des 15. und 16. Jahrhunderts und die Gesetzgebung des Konzils von Trient schnitten die iurisdictio delegata zurück, was auch zu einem Rückgang der „Justizbriefe“ führte. 55

Ein weiterer Weg, der zur Ausweitung der päpstlichen Gerichtsbarkeit führte, war die Einführung der Gerichtsbarkeit der päpstlichen Nuntien. Die römische Kurie hatte im 16. Jahrhundert auf dem Boden des Heiligen Römischen Reiches zwei Ständige Nuntiaturen errichtet,

\footnotetext{
${ }^{54}$ MÜLLER, Päpstliche Delegationsgerichtsbarkeit; HAGENEDER, Die geistliche Gerichtsbarkeit; DERS., Zur Effizienz der römischen Kurie; HERDE, Zur päpstlichen Delegationsgerichtsbarkeit; NÖRR, Romanischkanonisches Prozessrecht 10-14.

${ }^{55}$ HeRDE, Audientia 177f.; DERS., Zur päpstlichen Delegationsgerichtsbarkeit 40 .
}

eine in Wien (seit 1529), die andere in Köln (seit 1584). Sehr viel später wurde eine weitere Nuntiatur in München (seit 1785) gegründet. Die päpstlichen Nuntien waren in erster Linie als diplomatische Vertreter des Heiligen Stuhls tätig, doch beanspruchten sie daneben auch eine eigene Jurisdiktion. Diese Nuntiaturgerichte standen einerseits in Konkurrenz zu den ordentlichen Gerichten der Kirchenprovinzen und der römischen Kurie, anderseits zu den Gerichtshöfen der Fürstentümer und zu den Gerichten des Heiligen Römischen Reiches. Dies führte nicht selten zu heftigen Kompetenzkonflikten, wenn die Nuntien Appellationen gegen erstinstanzliche Urteile deutscher Gerichte behandelten. Streitigkeiten um die Zuständigkeit der Gerichte gab es vor allem dann, wenn die causa im Grenzgebiet zwischen weltlichem und geistlichem Recht lag. So wurde nicht selten in Benefizialsachen darum gekämpft, ob ein weltliches oder ein kirchliches Gericht zuständig sei. Gerade in den drei geistlichen Kurfürstentümern des Alten Reiches häuften sich die Streitfälle. ${ }^{56}$ Die Kompetenzproblematik war so bedeutsam, dass sie im Abschied des Reichstages zu Regensburg vom 17. Mai 1654 (Jüngster Reichsabschied, Art. 164) angesprochen wurde. ${ }^{57}$ Damit war der Kompetenzstreit zum Gegenstand des Reichsverfassungsrechts geworden: Es galt, die Kompetenz von Reichskammergericht und Reichshofrat zu wahren. In den Wahlkapitulationen der Kaiser wird der Standpunkt der weltlichen Seite fortgeschrieben, so auch in Art. 19 der Wahlkapitulation von Kaiser Leopold I. von 1658. Gegen diesen Versuch, die Ausübung päpstlicher Jurisdiktionsrechte durch die apostolischen Nuntien zu beschränken, erhob der päpstliche Nuntius Giuseppe Maria

\footnotetext{
${ }^{56}$ Hierzu BECKER, Die Sacra Rota Romana 15-18. Grundlegend nun OESTMANN, Geistliche und weltliche Gerichte, insbes. 123-229.

57 Text bei Buschmann, Kaiser und Reich 250f.
} 
Sanfelice (Nuntius in Köln 1652-1659) Protest. ${ }^{58}$ Gleichwohl hielt man im Reich an dem eigenen Standpunkt fest, wie die Texte des Art. XIV der kaiserlichen Wahlkapitulationen seit Karl VI. (1711) belegen. In der genannten Wahlkapitulation von 1711 heißt es:59,$[\ldots]$ gleichergestalt wollen Wir, wenn es sich etwan begäbe, daß die causae civiles von ihrem ordentlichen weltlichen Gericht im Heiligen Röm. Reich ab- und ausser dasselbe ad Nuntios apostolicos, oder wol gar ad Curiam romanam gezogen würden, solches abschaffen, vernichten und ernstlich verbiethen, auch Unserem Kaiserlichen Fiskalen, so wohl an Unserm Kayserlichen Reichs-Hof-Rath, als CammerGericht anbefehlen, wider diejenige, so wohl Partheyen als Advokaten, Prokuratoren und Notarien, die sich hinführo dergleichen anmassen und darinn einiger Gestalt gebrauchen lassen würden, mit behöriger Anklag von Amts wegen zuverfahren, damit die Ubertreter dem nechsten gebührend angesehen, und bestrafft werden mögen $[\ldots]^{\prime \prime}$.

Von Seiten der Bischöfe und Metropoliten ging es darum, ihre ortskirchliche Jurisdiktion gegen den Anspruch der Nuntiaturgerichte zu verteidigen. Dies zeigte sich insbesondere in den Koblenzer Gravamina ${ }^{60}$ von 1769 und im bekannten Nuntiaturstreit, ${ }^{61}$ der ab 1785 die Verfassung des Heiligen Römischen Reiches erschütterte (Emser Punktation $^{62}$ von 1786).

\footnotetext{
${ }^{58}$ Abdruck von Art. 19 der kaiserlichen Wahlkapitulation von 1658 und des Protests des Nuntius vom 9. 8. 1658 bei FELDKAMP, Studien und Texte 148-153.

${ }^{59}$ Zit.n. Ihro Röm. Kays. Maj. CAROLI VI WahlCapitulation 29f. Die Bestimmung in dem Entwurf einer beständigen Wahlkapitulation von 1711, Art. XIV, bei BusCHMANN, Kaiser und Reich 293-295. Vgl. hierzu OEstmanN, Geistliche und weltliche Gerichte $163 \mathrm{ff}$.

${ }^{60}$ FEINE, Kirchliche Rechtsgeschichte 569f.; STEINRUCK, Koblenzer Gravamina.

${ }^{61}$ FEINE, Kirchliche Rechtsgeschichte 569ff.; STEINRUCK, Nuntiaturstreit.

${ }^{62}$ STEINRUCK, Emser Kongreß.
}

\section{Zur Vorbildfunktion der kirchlichen Appellationsordnung im weltlichen Prozess}

Es ist eine bekannte Erscheinung, dass das kanonische Recht die Entwicklung des weltlichen Rechts beeinflusst hat. ${ }^{63}$ Im Hinblick auf das Prozessrecht der päpstlichen Dekretalen ist dies besonders deutlich. So wundert es nicht, dass auch in den Territorien, die sich der Reformation zugewandt hatten, das kanonische Prozessrecht nach wie vor eine große Rolle spielte. ${ }^{64}$ Die Kommentare zum kanonischen Prozess, also insbesondere die Glossen $\mathrm{zu}$ den Texten des jeweils zweiten Buches des Liber Extra, des Liber Sextus und der Clementinae, wurden für die Ausgestaltung des Prozessrechts in allen europäischen Territorien im 16. und 17. Jahrhundert herangezogen. Das Gleiche kann man über die großen Handbücher der Kanonistik sagen, in denen das Recht der Appellation dargestellt worden war. Deshalb sollen an dieser Stelle die Autoren der wichtigsten Prozesstraktate genannt werden, in denen das Recht der Appellation ausführlich dargestellt worden ist. Alle genannten Handbücher haben jeweils viele Auflagen erlebt, was ihren Erfolg in der Praxis belegt. Im Folgenden sind vorzugsweise jene Auflagen genannt, von denen es digitalisierte Ausgaben gibt, die über das Internet leicht zugänglich sind:

- BREDERODE, Tractatus de appellationibus

- DuRANDI, Speculum iuris (Lib. II, Partic. III: De appellationibus, 827-882)

- LANCELOTTUS, Institutiones iuris canonici

- Luca, De appellatione

- MARANTA, Speculum aureum

\footnotetext{
63 Trusen, Anfänge; Wolter, Ius canonicum; LANDAU, Die Anfänge der Prozessrechtswissenschaft; BERMAN, Recht und Revolution; BELLOMO, Europäische Rechtseinheit, insbes. 77-79.

${ }^{64}$ BurmeIster, Das Studium der Rechte 90-93; HelmHOLZ, Roman canon law; DERS., Canon law.
} 
- Pirhing, Ius canonicum

- Reiffenstuel, De appellationibus, recusationibus et relationibus, Tit. $28 \S \S 1-12$, 489526.

- RIDOLFINI, De ordine procedendi

- SCACCIA, Tractatus de sententia

- Ders., Tractatus de appellationibus

- Schmalzgrueber, Ius ecclesiasticum universum

- WAMESIUS, Recitationes

- DERS., Tractatus de appellationibus.

Bezeichnend ist auch, dass man schon früh versuchte, das kanonische und das weltliche Prozessrecht rechtsvergleichend darzustellen. Als Beispiele seien genannt:

- KÖNIG, Processus und Practica und

- BÖHMER, De collectionibus. ${ }^{65}$

Peter Landau ${ }^{66}$ hat die Vorbildfunktion des kanonischen Rechts im Hinblick auf die mittelalterliche Entwicklung des weltlichen Prozessrechts mit folgenden treffenden Worten beschrieben: „Lange vor dem Auftreten rechtsgelehrter Richter in den weltlichen Gerichten wurde die Rechtsprechung der kirchlichen Gerichte bereits durch wissenschaftlich geschulte Offiziale ausgeübt, so dass die Gerichtsverfassung der Kirche zum Vorbild für das weltliche Gerichtswesen werden konnte. Die Gerichtsverfassung mit so grundlegenden Instituten wie einem umfassenden Appellationsrecht, das die Einheitlichkeit der Rechtsanwendung verbürgte, einem Instanzenzug und der Organisation von zentralen obersten Kollegialgerichten an der päpstlichen Kurie hat zum erstenmal ein europäisches Modell einheitlicher Gerichtsorganisation entstehen lassen". Diese Bewertung gilt genauso für die frühe Neuzeit.

\footnotetext{
${ }^{65}$ Als Vorwort zu: MeviUs, Decisiones.

${ }^{66}$ LANDAU, Der Einfluß des kanonischen Rechts, insbes. 48
}

\section{Korrespondenz:}

Prof. em. Dr. Hans-Jürgen Becker

Universität Regensburg

Juristische Fakultät

Bürgerliches Recht, Europäische Rechtsgeschichte und Kirchenrecht

Universitätsstr. 31, 93053 Regensburg, Deutschland

hans-juergen.becker@jura.uni-regensburg.de

\author{
Abkürzungen: \\ C. Causa \\ can. canon, canones \\ Lib. Liber \\ Partic. Particula \\ q. quaestio \\ sess. sessio \\ Tit. Titulus
}

Siehe auch das allgemeine Abkürzungsverzeichnis: http://www.rechtsgeschichte.at/files/abk.pdf

\section{Literatur:}

Josephus Alberigo u.a. (Hgg.), Conciliorum oecumenicorum decreta (Bologna ${ }^{3} 1973$ ).

A. Amanieu, Art. Appel, in: Raoul NaZ u.a. (Hgg.), Dictionnaire de droit canonique, Bd. 1 (Paris 1935) 764-807.

Hans-Jürgen BECKER, Die Appellation vom Papst an ein allgemeines Konzil. Historische Entwicklung und kanonistische Diskussion im späten Mittelalter und in der frühen Neuzeit (= Forschungen zur kirchlichen Rechtsgeschichte und zum Kirchenrecht 17, Köln-Wien 1988).

DERS., Kardinal Giovanni Battista de Luca (1613-1683) und die Sacra Rota Romana, in: ZRG KA 126 (2009) 313-340.

DERS., Die Sacra Rota Romana in der frühen Neuzeit, in: Leopold AUER, Werner OGRIS, Eva ORTLIEB (Hgg.), Höchstgerichte in Europa. Bausteine frühneuzeitlicher Rechtsordnungen (= Quellen und Forschungen zur höchsten Gerichtsbarkeit im Alten Reich 53, Köln-Weimar-Wien 2007) 1-18.

Manlio BELlOMO, Europäische Rechtseinheit. Grundlagen und System des Ius Commune (München 2005).

Harold J. BERMAN, Recht und Revolution. Die Bildung der westlichen Rechtstradition (Frankfurt am Main 1991). 
BERNHARD VON ClAIRVAUX, De consideratione libri V ad Eugenium III., Lib. III, 2.10, in: Jacques Paul Migne (Hg.), Patrologia Latina, Bd. 182 (Paris 1854) 728-808.

Justus Henning BÖHMER, De collectionibus, usu et auctoritate decisionum, praecipue Rotae Romanae et Mevianarum (Frankfurt am Main 1740) (als Vorwort zu: David MeviUs, Decisiones super causis praecipuis ad praedictum tribunal Regium delatis (Frankfurt am Main ${ }^{71740)}$ ).

Pieter Cornelis van BREDERODE, Tractatus de appellationibus (Frankfurt am Main 1592).

Hanns Christof BRENNECKE, Rom und der dritte Kanon von Sardika, in: ZRG KA 69 (1983) 15-45.

Karl Heinz BurmeIster, Das Studium der Rechte im Zeitalter des Humanismus im deutschen Rechtsbereich (Wiesbaden 1974).

Arno Buschmann (Hg.), Kaiser und Reich. Verfassungsgeschichte des Heiligen Römischen Reiches Deutscher Nation vom Beginn des 12. Jahrhunderts bis zum Jahre 1806 in Dokumenten, Teil 2 (Baden-Baden ${ }^{21994) .}$

Ihro Röm. Kays. Maj. CAROLI VI Wahl-Capitulation und Reversales cum notis differentiam a Project. Capitulat. Perpetuae ibique indicantibus (o.O. 1741).

Gundula CASPARY, Späthumanismus und Reichspatriotismus. Melchior Goldast und seine Editionen zur Reichsverfassungsgeschichte (= Formen der Erinnerung 25, Göttingen 2006).

Heinrich DANNENBAUER, Ein deutscher Reformantrag vom Konzil zu Ferrara 1438, in: Historisches Jahrbuch 70 (1951) 106-114.

Guilelmus DuRANDI, Speculum iuris, Bd. 1 (Basel 1574, ND Aalen 1975).

Michael DuRST, Art. Sardika 2, in: LThK³, Bd. 9 (Freiburg im Breisgau 1993) 71f.

Jacobus EMERIX, Tractatus seu notitia S. Rotae Romanae (ca. 1677), hg. v. Charles Lefebvre (= Monumenta christiana selecta 8, Tournai [1960]).

Wilhelm EndEMANN, Civilprozessverfahren nach der kanonistischen Lehre, in: Zeitschrift für deutschen Civilprozess 15 (1891) 177-326.

Hans Erich FEINE, Kirchliche Rechtsgeschichte: Die katholische Kirche (Köln-Graz $\left.{ }^{4} 1964\right)$.

Michael F. FELDKAMP, Studien und Texte zur Geschichte der Kölner Nuntiatur, Bd. 2 (= Collectanea Archivi Vaticani 31, Rom 1993).

Lucius FERRARIS, Art. Appellatio, in: DERS., Prompta bibliotheca canonica, polemica, rubristica, historica, Bd. 1 (Paris 1863) 577-658.
Linda FoWLER-MAgerL, Ordo iudiciorum vel ordo iudiciarius (Ius commune Sonderhefte 19, Frankfurt am Main 1984).

Emil Friedberg (Hg.), Corpus iuris canonici, Bd. 1-2 (Leipzig 1879).

Pietro Gasparri (Hg.), Codex iuris canonici Pii X Pontificis Maximi iussu digestus Benedicti Papae $\mathrm{XV}$ auctoritate promulgatus praefatione, fontium annotatione et indice analytico-alphabetico (Rom 1918).

Klaus Martin GIRADET, Appellatio. Ein Kapitel kirchlicher Rechtsgeschichte in den Kanones des 4. Jahrhunderts, in: Historia. Zeitschrift für Alte Geschichte 23 (1974) 98-127.

Melchior GOLDAST, Monarchia S. Romani Imperii sive Tractatus de iurisdictione imperiali, regia et pontificia seu sacerdotali, Bd. 2 (Frankfurt am Main 1613).

Othmar HAGENEDER, Die geistliche Gerichtsbarkeit in Ober- und Niederösterreich (= Forschungen zur Geschichte Oberösterreichs 10, Graz-Wien-Köln 1967).

DERS., Zur Effizienz der römischen Kurie als Gerichtshof um 1200, in: Wilfried Ehbrecht u.a. (Hgg.), Der weite Blick des Historikers. Einsichten in Kultur-, Landes- und Stadtgeschichte. Peter Johanek zum 65. Geburtstag (Köln-Weimar-Wien 2002) 99-112.

Carl Joseph von HefEle, Conciliengeschichte, Bd. 2 (Freiburg im Breisgau ${ }^{21875), ~ B d . ~} 7$ (ebd. 1874).

Richard Helmholz (Hg.), Canon Law in Protestant Lands (= Comparative Studies in Continental and Anglo-American Legal History 11, Berlin 1992).

DERs., Roman Canon Law in Reformation England (=Cambridge Studies in English Legal History, Cambridge 1992).

Peter HeRDE, Audientia litterarum contradictarum. Untersuchungen über die päpstlichen Justizbriefe und die päpstliche Delegationsgerichtsbarkeit vom 13. bis zum Beginn des 16. Jahrhunderts, Bd. 1 (= Bibliothek des Deutschen Historischen Instituts in Rom 31, Tübingen 1970).

DERS., Zur päpstlichen Delegationsgerichtsbarkeit im Mittelalter und in der frühen Neuzeit, in: ZRG KA 88 (2002) 20-43.

Philipp HeRgENRÖTHER, Die Appellationen nach dem Decretalenrechte (Eichstätt 1875).

Paul HinschiUs, Das Kirchenrecht der Katholiken und Protestanten in Deutschland. System des katholischen Kirchenrechts mit besonderer Rücksicht auf Deutschland, Bd. 1 (Berlin 1869).

Rudolf HOKE, Art. Goldast, Melchior (1578-1653), in: $\mathrm{HRG}^{2}$, Bd. 2 (Berlin 2009) 446-447. 
Erwin JACOBI, Der Prozeß im Decretum Gratiani und bei den ältesten Dekretisten, in: ZRG KA 3 (1913) 223-343.

Stefan KILlERMANN, Die Rota Romana. Wesen und Wirken des päpstlichen Gerichtshofes im Wandel der Zeit (= Adnotationes in ius canonicum 46, Frankfurt am Main 2009).

Chilian KÖNIG, Processus und Practica der Gerichtsleuffte nach sechsischem Gebrauch, aus Bepstlichen, Keiserlichen und Sechsischen Rechten (Wittenberg 1541).

Iohannes Paulus LANCELOTTUS, Institutiones iuris canonici (Venedig 1598). Weitere Ausgabe: Lyon 1661.

Peter LANDAU, Die Anfänge der Prozessrechtswissenschaft in der Kanonistik des 12. Jahrhunderts, in: Orazio Condorelli u.a. (Hgg.), Der Einfluss der Kanonistik auf die europäische Rechtskultur, Bd. 1 (= Norm und Struktur 37/1, Köln-Weimar-Wien 2009) 7-23.

DERS., Der Einfluß des kanonischen Rechts auf die europäische Rechtskultur, in: Reiner ScHUlzE (Hg.), Europäische Rechts- und Verfassungsgeschichte. Ergebnisse und Perspektiven der Forschung (= Schriften zur europäischen Rechts- und Verfassungsgeschichte 3, Berlin 1991) 39-57.

Susanne LEPSIUS, Art. Durantis, Guilelmus (um 12301296), in: $\mathrm{HRG}^{2}$, Bd. 1 (Berlin 2008) 1168-1170.

Wiesław LiTEwsKI, Appeal in Corpus Iuris Canonici, in: Annali di storia del diritto 14-17 (1974) 14521521.

DERS., Der römisch-kanonische Zivilprozeß nach den älteren ordines iudiciarii, 2 Bde. (Krakau 1999).

Johannes Baptista de LUCA, De appellatione, in: DERS., Theatrum veritatis et justitiae, Lib. 15 (Venedig 1726), discursus 37, 126-136.

Joannes Dominicus MANSI, Sacrorum conciliorum nova et amplissima collectio, Bd. 29 (Paris 1904, ND Graz 1961).

Roberto MARANTA, Speculum aureum et lumen advocatorum (Venedig 1557).

David Mevius, Decisiones super causis praecipuis ad praedictum tribunal Regium delatis (Frankfurt am Main ${ }^{71740)}$

Jürgen MiethKE, Lorenz WeINRICH (Hgg.), Quellen zur Kirchenreform im Zeitalter der großen Konzilien des 15. Jahrhunderts, Teil 1-2 (Darmstadt 1995-2002).

Anton MORHARD, Die gerichtliche Berufung im kanonischen Recht. Eine Analyse des klassischen remedium iuris (= Adnotationes in ius canonicum 1, Frankfurt am Main u.a. 1995).
Harald MÜLLER, Päpstliche Delegationsgerichtsbarkeit in der Normandie (12. und frühes 13. Jahrhundert), 2 Bde. (= Studien und Dokumente zur Gallia Pontificia 4, Bonn 1997).

Knut Wolfgang NÖRR, Ein Baustein der mittelalterlichen Rechtskirche: Die Appellatio extraiudicialis, in: Rosalius Iosephus CASTILLO LARA (Hg.), Studia in honorem Eminentissimi Cardinalis Alphonsi M. Stickler (= Studia et textus historiae iuris canonici 7, Rom 1992) 463-482.

DERS., Romanisch-kanonisches Prozessrecht. Erkenntnisverfahren erster Instanz in civilibus (Berlin 2012).

Peter Oestmann, Geistliche und weltliche Gerichte im Alten Reich. Zuständigkeitsstreitigkeiten und Instanzenzüge (= Quellen und Forschungen zur Höchsten Gerichtsbarkeit im Alten Reich 61, Köln-Weimar-Wien 2012).

Georg PHILLIPS, Kirchenrecht, Bd.5, Abt. 1 (Regensburg 1854).

Ehrenreich PIRHING, Ius canonicum in V libros decretalium (Dillingen 1645 u.ö.).

Willibald M. PLÖCHL, Geschichte des Kirchenrechts, Bd. 2 (Wien-München ${ }^{2} 1962$ ).

Richard PUZA, Res iudicata. Rechtskraft und fehlerhaftes Urteil in den Decisionen der Römischen Rota (= Grazer rechts- und staatswissenschaftliche Studien 29, Graz 1973).

DERS., Rescriptum und Commissio. Die Entscheidung der signatura iustitiae im 16. und 17. Jahrhundert, in: ZRG KA 66 (1980) 354-370.

DERS., Signatura iustitiae und commissio. Ein Beitrag zum Prozeßgang an der römischen Kurie in der Neuzeit, in: ZRG KA 64 (1978) 95-115.

Niccolò del RE, La Curia Romana. Lineamenti storicogiuridici (Rom ${ }^{4}$ 1998).

Anacletus REIFFENSTUEL, De appellationibus, recusationibus et relationibus, in: DERS., Ius canonicum universum, Bd. 2 (Antwerpen 1755).

Pietro RIDOLFINI, De ordine procedendi in iudiciis in Romana curia (Venedig 1680).

Fernando della RoccA, Il processo in Graziano, in: Studia Gratiana 2 (1954) 279-303.

Johannes Baptist SÄGMÜLLER, Lehrbuch des katholischen Kirchenrechts, Bd. 2 (Freiburg im Breisgau $\left.{ }^{3} 1914\right)$.

Sigismundo SCACCIA, Tractatus de appellationibus (Frankfurt am Main 1615 u.ö.).

DERS., Tractatus de sententia et re iudicata (Genf 1670).

Bernhard Schimmelpfennig, Das Papsttum. Von der Antike bis zur Renaissance (Darmstadt ${ }^{62009)}$. 
Alfred SchINDLER, Art. Afrika I, in: Theologische Realenzyklopädie, Bd. 1 (Berlin 1977) 640-700.

Steffen SCHLINKER, Die prozessuale Funktion der sententia interlocutoria im spätmittelalterlichen gelehrten Zivilprozess, in: ZRG KA 96 (2010) 152185.

Franz SCHMALzGRUEBER, Ius ecclesiasticum universum, 5 Teile (Ingolstadt 1728).

Heribert SCHMITZ, Appellatio extraiudicialis. Entwicklungslinien einer kirchlichen Gerichtsbarkeit über die Verwaltung im Zeitalter der klassischen Kanonistik (1140-1348) (= Münchener Theologische Studien 3, Kanonistische Abteilung 29, München 1970).

Franz Egon SCHNEIDER, Die Römische Rota nach geltendem Recht auf geschichtlicher Grundlage (= Veröffentlichungen der Görres-Gesellschaft zur Pflege der Wissenschaft im katholischen Deutschland 22, Paderborn 1914).

Josef STEINRUCK, Art. Emser Kongreß, in: LThK³ Bd. 3 (Freiburg im Breisgau-Wien 1995) 637f.
DERS., Art. Koblenzer Gravamina, in: LThK 3 , Bd. 6 (Freiburg im Breisgau-Wien 1997) 164.

DERS., Art. Nuntiaturstreit, in: LThK ${ }^{3}$, Bd. 7 (Freiburg im Breisgau-Wien 1998) 949.

STEPHAN VON TOURNAI, Summe über das Decretum Gratiani, hg. v. Johann Friedrich von ScHULTE (Gießen 1891).

Winfried TRUSEN, Anfänge des gelehrten Rechts in Deutschland. Ein Beitrag zur Geschichte der Frührezeption (= Recht und Geschichte 1, Wiesbaden 1962).

Walter UlLmanN, Kurze Geschichte des Papsttums im Mittelalter (Berlin 1978).

Johannes WAMESIUS, Recitationes ad tit. 28 libr. II decretalium De appellationibus (Löwen 1599).

DERS., Tractatus de appellationibus ad praxin forense accomodatissimus (Löwen 1600).

Udo WOLTER, Ius canonicum in iure civili. Studien zur Rechtsquellenlehre in der neueren Privatrechtsgeschichte (= Forschungen zur neueren Privatrechtsgeschichte 23, Köln-Wien 1975).

\section{Zusammenfassung}

Das mittelalterliche kanonische Recht hat mit der Ausbildung eines umfassenden Appellationsrechts und der Einrichtung eines geordneten Instanzenzuges bewirkt, dass sich das kanonische Recht überall in Europa durchsetzte und zugleich die Einheitlichkeit der Rechtsprechung gefördert werden konnte. Als seit dem 16. Jahrhundert die weltlichen Territorien dazu übergingen, ihr Gerichtswesen zu zentralisieren und die Rechtsprechung durch gelehrte Richter ausüben zu lassen, konnte man auf das System des kanonischen Prozessrechts und auf das entwickelte Institut der Appellation als Vorbild zurückgreifen. Allerdings entstanden nun auch Konflikte um die Zuständigkeit der jeweiligen Jurisdiktion: Nachdem im Heiligen Römischen Reich Ständige Nuntiaturen (Wien und Köln) eingerichtet worden waren, die - in Ergänzung der kurialen Gerichte der Rota Romana und der Signatura Apostolica - eine eigene Jurisdiktion beanspruchten, entstanden Kompetenzkonflikte mit dem Reichskammergericht und dem Reichshofrat.

\section{Summary}

Medieval canon law shaped a comprehensive right of appeal as well as a well-ordered hierarchy of judicial instances. It was thus able to establish itself throughout Europe and at the same time advance the homogeneity of the dispensation of justice. When, from the $16^{\text {th }}$ century onwards, secular territories started to centralize their judicature and to entrust the dispensation of justice to learned judges, they could look to canon procedural law and its well-developed mechanism of appeal for a model. This, however, engendered conflicts of jurisdiction. For once permanent nunciatures had been set up in the Holy Roman Empire (Vienna, Cologne) to supplement the papal courts of the Rota Romana and the Signatura Apostolica, and once these nunciatures claimed jurisdiction, conflicts emerged with both the Imperial Chamber Court ("Reichskammergericht") and the Imperial Aulic Council ("Reichshofrat"). 
